\title{
The Effects of Manufacturer's Prefit and Real-Ear Fitting on the Predicted Speech Perception of Children with Severe to Profound Hearing Loss
}

DOI: $10.3766 /$ jaaa. 16150

\author{
Tian Kar Quar* \\ Cila Umat* \\ Yong Yee Chew*
}

\begin{abstract}
Background: The use of probe microphone measures in hearing aid verification is often neglected or not fully used by practitioners. Some practitioners rely on simulated gain and output provided by manufacturer's fitting software to verify hearing aids.
\end{abstract}

Purpose: This study aims to evaluate the effectiveness of manufacturer's prefit procedure in matching the prescribed real-ear targets. It also aims to study its correlated impact on the predicted speech perception in children with severe and profound hearing loss.

Research Design: This cross-sectional experiment was carried out by measuring the output of hearing aids based on prefit versus real-ear at low-, moderate-, and high-input levels. The predicted speech perception for different hearing aid fittings was determined based on the Speech Intelligibility Index (SII).

Study sample: Sixteen children (28 ears) aged between 4 and $7 \mathrm{yr}$, with severe to profound sensorineural hearing loss took part in the study.

Method: Two different types of hearing aids (Phonak and Unitron) were programmed based on their respective manufacturers' Desired Sensation Levels (DSL) v5 Child procedure. The hearing aids were then verified using coupler-based measurements and individual real-ear-to-coupler differences. The prefit outputs were compared with the DSL v5 Child-prescribed outputs at low-, moderate-, and highinput levels. The hearing aids were then adjusted to closely match the prescribed output. The SIls were calculated for the fittings before and after adjustment.

Data Collection and Analysis: Sixty four percent of fittings that were based on the prefit procedure achieved the optimal fit-to-targets, with less than 5-dB RMS deviations from the DSL v5 Child targets. After adjusting the hearing aids to attempt to meet the DSL v5 Child targets, 75\% of the ears tested achieved the optimal fit-totargets. On average, hearing aid outputs generated by the manufacturer's prefit procedure had good and reasonable agreement with the DSL v5 Child-prescribed outputs at low- and mid-frequencies. Nonetheless, at $4000 \mathrm{~Hz}$, the hearing aid output mostly fell below the DSL v5 Child-prescribed outputs. This was still the case even after the hearing aid was adjusted to attempt to match with the targets. At low input level, some prefit outputs were found to be higher than the prescribed outputs. The deviations of prefit outputs from the prescribed outputs were dependent on the type of hearing aid and input levels. There was no significant difference between the SII calculated for fittings based on the prefit and adjusted fit.

Conclusions: Prefit procedure tends to produce outputs that were below the DSL v5 Child-prescribed outputs, with the largest mean difference at $4000 \mathrm{~Hz}$. Even though the hearing aid gains were adjusted to attempt to match with the targets, the outputs were still below the targets. The limitations of hearing aids to match the DSL v5 Child targets at high-frequency region have resulted in no improvement in the children's predicted speech perception.

Key Words: achieved gain, fit-to-targets, initial-fit, manufacturer fitting, prescribed gain, real-ear measures

${ }^{*}$ School of Rehabilitation Sciences, Faculty of Health Sciences, Universiti Kebangsaan Malaysia, Kuala Lumpur, Malaysia 


\begin{abstract}
Abbreviations: ANOVA = analysis of variance; $\mathrm{DSL}=$ desired sensation level; $\mathrm{FL}=$ frequency lowering; $\mathrm{HLD}=$ hearing loss desensitization; HTL = hearing threshold levels; NAL = National Acoustic Laboratories; NAL-NL1 = NAL, Nonlinear, version 1; REAR = real-ear aided response; RECD = real-ear to coupler difference; RMS = root-mean-square; SD = standard deviation; SII = speech intelligibility index; SPL = sound pressure level
\end{abstract}

\section{INTRODUCTION}

$\mathrm{A}$ prescriptive approach to hearing aid fitting is based on hypothesized relationships between the characteristics of hearing-impaired individuals and the amplification characteristics they require for optimal auditory function (Byrne, 1983). Numerous prescriptive procedures have been developed for fitting hearing aids to individuals with hearing impairment. The National Acoustic Laboratories (NAL) and the Desired Sensation Level (DSL) procedures are two examples of generic prescriptive methods that are relied on by many clinicians on hearing aids fitting (Mueller and Picou, 2010). Many studies had been conducted in the past to test the validity of these procedures in adult (Mueller, 2005a) and in pediatric (Ching et al, 2010; Quar et al, 2013; McCreery et al, 2015; Bagatto et al, 2016). According to Seewald et al (1985), infants and children are most likely to rely on hearing aid fits with methods based on prescriptive approach. This is because they are usually neither reliable in clinical tests nor can they provide feedback to assist clinicians in tuning the hearing aids. Hence, it is important to verify hearing aids to ensure the real-ear gain/output of hearing aids worn by children meet the prescriptive targets, and the maximum output does not exceed the prescribed levels.

The American Academy of Audiology Clinical Practice Guidelines (AAA, 2013) suggests two options for hearing aid verification in children. One, real-ear aided response (REAR) that measures the sound pressure level (SPL) in the child's ear (in situ) using a probe microphone while the hearing aid is turned on. It should be noted that valid and reliable in situ probe microphone measurements are often difficult to obtain from children. This is due to typical restlessness among infants and young children that causes difficulties in obtaining the measurements. Also, the difficulty is caused by their uneasiness with the presence of the probe tube in their ear canals during the fine tuning process. Apart from REAR, simulated REAR measurements in the coupler using measured or age-appropriate real-ear to coupler difference (RECD) can also be employed. In this approach, RECD is used to convert coupler measures to SPL estimates in the child's ear. It is also used to convert individual's hearing thresholds from dB HL to dB SPL in the ear canal. This allows all audiometric and electroacoustic variables in a common reference level which is the ear canal SPL (Scollie et al, 2005). Moreover, past studies reveal that the RECD procedure is repeatable and can be used to accurately predict real-ear aided performance (Seewald, 1991; Moodie et al, 1994; Sinclair et al, 1996; Seewald et al, 1999).

McCreery et al (2013) reported that about $86 \%$ of audiologists used either in situ real ear or RECD as their primary method of hearing aid verifications. A recent study by Bagatto et al (2016) examined the hearing aid outcomes of 115 children from four different clinics enrolled in the Infant Hearing Program in Ontario. The study concluded that well-fitted hearing aids (i.e., gain frequency-response of hearing aids verified according to the DSL v5 Child targets) would correlate with good auditory development milestones for the children's age. Furthermore, studies by McCreery et al (2015), Moeller and Tomblin (2015), and Tomblin et al (2015) found that well-fitted hearing aids contributed to good audibility of speech or speech intelligibility index (SII). The SII has been used successfully to predict speech perception ability for people with normal hearing (Pavlovic et al, 1986; Studebaker et al, 1997; Ching et al, 1998). Meanwhile, for individuals with hearing loss, Scollie (2008) reported that the SII could successfully be used to predict speech recognition scores. Nonetheless, the effects of age and hearing loss should be included in the development of a transfer function. This agrees with other studies that demonstrated SII as significant predictors for vocabulary development in children (Stiles et al, 2012) and for aided speech perception ability for adults and children with severe to profound hearing loss (Davidson and Skinner, 2006). Therefore, SII is deemed as an important element in the verification procedure and hence has been incorporated into the American Academy of Audiology (AAA, 2013) hearing aid fitting protocol.

Despite the clinical importance of hearing aid verification, Mueller and Picou (2010) found that nearly 50\% of audiologists and hearing instrument specialists in the United States who had access to probe-microphones did not use it routinely to match real-ear gain to gain prescribed. The percentage of audiologists that used real-ear measures was lower in their study, compared with what was reported by McCreery et al (2013). This may be due to different participant group-McCreery et al focused on audiologists who were involved in only pediatric cases. Also, the emphasis on adhering to the standard guidelines for pediatric amplification at the study sites may also contribute to those differences. According to Mueller (2005b), there are several reasons why the use of real-ear measures in hearing aid verification is often neglected or not fully used by practitioners. Some of the main reasons 
are lack of understanding on the correlation between verification with hearing aid satisfaction, the assumption that verification is not suitable for hearing aids with advanced features, poor training programs, and lack of professional and personal dedication in performing the real-ear measures. In addition, the development of fitting software by manufacturers may also be a reason. Most of today's fitting software provides simulated gain and output for 2-cc coupler, ear simulator, real-ear insertion gain, and REAR. Since these simulated values are provided, practitioners may accept these in their fitting process at face values without any knowledge of the true in situ hearing aid response (Mueller, 2001; 2005b; Hawkins and Cook, 2003).

Hawkins and Cook (2003) conducted a study on 12 adults with sensorineural hearing loss to compare the simulated insertion gain provided by manufacturers and the actual insertion gain measured from participants. The results showed that in the frequency region of 250$1000 \mathrm{~Hz}$, the differences between the simulated and actual insertion gain were within $\pm 5 \mathrm{~dB}$. In the higher frequency region, however, the differences were higher and the actual insertion gain values were less than the simulated values ( $>10 \mathrm{~dB}$ difference at $4000 \mathrm{~Hz}$ ). Another study conducted by Aarts and Caffee (2005) supported the finding. They examined the accuracy of one manufacturer's fitting software in predicting the REAR on adults. In their study, one hearing aid model was used to measure REAR from 79 ears. The values were then compared with simulated REAR provided by fitting software under four conditions: two hearing loss configurations and two input levels. The end results demonstrated that the mean of actual REAR values exceeded the predicted REAR values from 500 to $1000 \mathrm{~Hz}$ and above $4000 \mathrm{~Hz}$, and fell short on predicted REAR values for frequencies between 1500 and $4000 \mathrm{~Hz}$. It was explained that the poor predictability of actual REAR values was caused by the average transforms used by the fitting software to generate the estimates of the hearing aid output in the ear.

It should be noted that the two studies described previously compared the simulated gain displayed on manufacturer's fitting software/screen with the actual gain measured in the ear canal. The studies did not compare the gain generated by the manufacturer's fitting software with the gain prescribed by a prescriptive procedure in the ear canal. This should not be taken for granted as some clinicians might choose one prescriptive procedure (e.g., DSL v5) offered by the manufacturer's fitting software without verifying the hearing aids in the ear canal. In Aazh and Moore (2007), four types of digital hearing aids were programmed based on the NAL-Non-Linear, version 1 (NAL-NL1) procedure selected in the manufacturers' fitting software. This fitting procedure is referred to as "prefit" procedure. Real-ear-insertion-gain measurement was conducted on 42 ears using hearing aids that were programmed with prefit procedure. The prefit gain and
NAL-NL1 targets in the real ear were assessed, and the hearing aids were adjusted to match the targets if necessary. The results demonstrated that $64 \%$ of the prefit failed to come within $\pm 10 \mathrm{~dB}$ of the NAL-NL1 prescriptive targets at one or more frequencies between 250 and 4000 $\mathrm{Hz}$. After adjusting the hearing aids gain, $83 \%$ of the fittings achieved the pass criteria in matching with the NAL-NL1 targets. The study concluded that it is crucial to use real-ear measures to achieve accurate fittings.

The main purpose of this study was to examine how much prefits based on manufacturer-specific prescriptions (i.e., DSL v5 Child) deviate from the output prescribed by DSL v5 Child in children's ear canals. The ear-canal responses were predicted in the coupler using the RECD values measured from each child. There are several reasons on focusing on children with severe to profound hearing loss. First, most of previous studies focused on adults with mild to moderately severe hearing loss. For example, in Aazh and Moore (2007) study, hearing aids were programmed based on a manufacturer's prefit procedure and then compared with the NAL-NL1 prescribed gain in the real ear. The NAL procedure has a different fundamental in rationale from the DSL procedure which results in some differences in prescribed gains between the two procedures. The gain differences are the greatest when the hearing loss is severe to profound (Ching et al, 2015). Therefore, findings from previous studies that used the NAL-NL1 procedure could not be directly applied to fittings based on DSL v5 Child procedure. Likewise, Seewald et al (2008) found significant differences between simulated real-ear saturation response and gain frequency-response in a 6-mo old infant. These differences were resulted by different manufacturers' prescriptions. Moreover, different simulated real-ear output also resulted in substantial differences on the aided SII values among the manufacturers. The second reason is that it is possible that the mismatch between the gain predicted by fitting software, and the actual gain in children's ears is relatively higher than adults. This may be caused by biologic variations of the physical ear properties. This is evidenced by a longitudinal study by McCreery et al (2015). They found that children's ear canal acoustics changes over time, and the sound level in the ear canal decreases as age increases. These cause sound levels in the real ear become even harder for pediatric population to predict. Therefore, the present study aims to answer the following questions: (a) how much does the output of hearing aid that is fitted based on manufacturer-specific prescriptions deviate from the output prescribed by DSL v5 Child in real ear, for children with severe to profound hearing loss?; (b) can prefit hearing aids be adjusted to match the DSL v5 Child prescribed output across the test frequencies?; and (c) do hearing aids adjusted to closely match the DSL v5 Child prescriptions have better SII values than SII calculated based on prefit prescription? 


\section{METHOD}

\section{Participants}

This was a cross-sectional experiment in which the data were collected from 16 children (28 ears) aged 4$7 \mathrm{yr}$. Using the $\mathrm{F}$ family of tests in $\mathrm{G}$ Power analysis, this sample size is adequate in detecting a difference of 0.36 population standard deviation (SD), with 0.80 power at an error rate of $5 \%$. The participants were hearing-impaired children who attended the Audiology and Speech Sciences Clinic, Universiti Kebangsaan Malaysia, Kuala Lumpur. Only children with normal outer ear, with no excessive ear cerumen and middle ear pathology were reruited for the study. All participants had sensorineural hearing losses that ranged from severe to profound (mean hearing level at three frequencies [3FA] = $100 \mathrm{~dB}$ HL, SD = 14.5). The study was approved by the Human Ethics Committee of Universiti Kebangsaan Malaysia (UKM 1.5.3.5/244/NN-152-2014) and an informed consent was obtained from the parents of all participants.

\section{Hearing Assessment and RECD Measurement}

Otoscopic examination and tympanometry were carried out on all children to rule out any outer- and middle-ear anomalies. Play audiometry using insert earphones, Etymotic Research ER-3A was conducted to test the hearing levels at 250, 500,1000,2000, and $4000 \mathrm{~Hz}$ in a soundtreated room. The hearing test showed that nine of the ears had severe hearing loss (mean $3 \mathrm{FA}=83 \mathrm{~dB} \mathrm{HL}$, $\mathrm{SD}=9.2 \mathrm{~dB}$ ), and 19 of the ears had profound hearing loss $(3 \mathrm{FA}=108.8 \mathrm{~dB} \mathrm{HL}, \mathrm{SD}=9.5 \mathrm{~dB})$.

RECD was measured using the Audioscan Verifit RM500SL on the test ears. Prior to this, probe-tube calibration was carried out with regard to Audioscan Verifit (Audioscan, 2014) protocol. To obtain the RECD values, response of the 2-cc coupler was first measured by attaching the RECD transducer to the HA2-coupler in the text box. A thin probe tube was inserted $20 \mathrm{~mm}$ (4- to 5-yr old) and $25 \mathrm{~mm}$ (6- to 7-yr old) past the intertragal notch of participants for real-ear measurements (Moodie et al, 1994). A foam tip was also inserted into the ear until the most lateral portion of the foam tip was flushed with the opening of the participant's ear canal (Bagatto et al, 2006). This was done with care to ensure that the probetube remained at the same location in the ear canal. The same signal used for the coupler measurement was delivered through the foam tip to obtain the RECD values. Negative RECD values in the range of -1 to $-9 \mathrm{~dB}$ SPL at 250,500 , and/or $750 \mathrm{~Hz}$ was associated with acoustic leakage of stimuli from the auditory canal (Bagatto, 2001). In this case, the measurement was repeated using the same or larger foam tip, as precaution.

The present study deviates from the practice methods that recommends the use of a personal earmold, instead of an insert foam tip to measure hearing threshold levels (HTL) and RECD (AAA, 2013). Personal earmold is preferred in RECD and audiometry measures because in this case, coupling to the ear will match throughout the hearing aid fitting process. This allows the individual RECD to be used to correct thresholds and coupler hearing aid response to ear canal SPL (Bagatto et al, 2005). In addition, such practice provides a more accurate prediction of real-ear frequency response of hearing aids, as it takes into account the depth of earmold insertion, length of earmold sound bore, and acoustic leakage estimation of earmold versus insert earphones (Munro and Hatton, 2000). Nonetheless, insert foam tip was still employed to simulate the common practice at the research site. Also, personal earmold is not always feasible, especially if the earmold is not yet available or is outgrown (Moodie et al, 2016).

\section{Hearing Aid Programming}

Two hearing aids, Unitron Max 6 SP BTE and Phonak Naida Q30 UP were chosen to be used in this study. They were selected by the following requirments: (a) based on the manufacturer specifications, they provide gain up to $82 \mathrm{~dB}$ and output up to $142 \mathrm{~dB}$ SPL. They also offer fitting ranges within specifications that are suitable for severe to profound hearing losses; (b) both hearing aids have similar technology features-Unitron 6 SP has six channels and Phonak Naida has eight channels; (c) DSL v5 Child prescription is available in both manufacturers' fitting software. The electroacoustic charaterisitcs of both hearing aids were measured in a test box and compared with the respective manufacturer's data sheets to ensure good functionality.

The hearing aids were programmed using their respective manufacturer's fitting software that was installed in NOAH 3.0 software paltform. First, the participants' HTLs were keyed into the NOAH. Then, the fitting software generated the prefit based on the following features: size 13 tubing, occluded earmold, no vent, and all the advanced features deactivated (e.g., noise reduction system, frequency lowering [FL], and adaptive directional microphone). The predicted RECD values from the respective fitting software were used to program the hearing aid. The predicted RECD values were used and not the individual RECD values to create and maintain the original manufacturer's prefit program. Apart from that, the DSL v5 Child prescriptive formula was selected in the prefit program. The acclimatization level was set at a level (indicated as $100 \%$ on fitting software) that corresponds to the best match with the selected prescriptive targets.

\section{Hearing Aid Verification}

The participants' HTLs and individual RECD values were computed into the Audioscan Verifit system to 
calculate the DSLv5 Child targets. In the DSLv5 Child applications, participant's age and transducer insert foam-tip used to measure HTL were incorporated into the calculation of prescriptive targets. The outputs of hearing aids that have been programmed according to the prefit procedure were then measured with a HA2-coupler at 50, 65, and $75 \mathrm{~dB}$ SPL input levels, using a speech signal. The prefit outputs and target values generated by the DSL v5 Child procedure at 250, 500, 1000,2000 , and $4000 \mathrm{~Hz}$ for both hearing aids at three input levels were recorded for comparison. The prefit outputs of the hearing aids were then adjusted to attempt to meet the DSL v5 Child targets (within \pm 5 $\mathrm{dB}$ ) for the three input levels. It should be noted that the $\pm 5 \mathrm{~dB}$ tolarance level was based on the recommendation of the modernization of hearing aid services in the United Kingdom (Aazh and Moore, 2007). The measured output at each frequency was matched to the DSL v5 Child targets at a $65-\mathrm{dB}$ input level, followed by 50 and 75-dB SPL input levels. Subsequent to hearing aid adjustments at each input level, outputs measured at the preceding input level were cross-checked to make sure that the previous values did not change. If change that caused errors exceeding $5 \mathrm{~dB}$ of the target at any frequency between 250 and $4000 \mathrm{~Hz}$ was observed, further adjustments were made. More priority was given to match the DSL v5 Child targets at $65-\mathrm{dB}$ input level, followed by the 50 - and 75-dB input levels.

\section{SII}

The SII, which is a modification of the Articulation Index, is one of the methods used to predict speech intelligibility from a specified loudness level or degree of audibility (Moore and Glasberg, 1997; Ching et al, 2001). Determining aided SII will allow the estimation of the amount of audible signal above the hearing threshold and to ensure adequate audibility is received by the child (Ching et al, 2001). Note also that the aided SII normative values by degree of hearing loss are available to help clinicians estimate the quality of hearing aid fitting without needing to provide the aided output
(Bagatto et al, 2016). The aided SII values in this study were calculated by the Audioscan Verifit software for 50-, 65-, and 75-dB input levels. The software calculated the values by using the $1 / 3$ octave band method of ANSI S3.5-1997 without the $160 \mathrm{~Hz}$ band. It also does so without masking effects, and the results were expressed as a $\%$ by multiplying with 100 . Level distortion effects were included but no hearing loss desensitization (HLD) was applied (Audioscan Verifit, 2014). The SII values calculated for prefit outputs before and after hearing aid was adjusted to meet the DSL v5 Child targets at different input levels were recorded for data analysis.

\section{RESULTS}

\section{Fit-to-Targets Achieved by Prefit Procedure}

The root-mean-square (RMS) was calculated and observed at four frequencies $(500,1000,2000$, and 4000 $\mathrm{Hz})$ to assess if the manufacturer's prefit output deviated from the DSL v5 Child targets. RMS $<5 \mathrm{~dB}$ was considered to be an optimal fit-to-target that was achieved by the prefit procedure (McCreery et al, 2013; Ching et al, 2015). With regard to these criteria, $73 \%$ of the ears that were fitted with the prefit procedure deviated from the DSL v5 targets by more than $5 \mathrm{~dB}$ RMS. In other words, they failed to achieve the optimal fit-totargets. After adjusting the hearing aids to attempt to match with the DSL v5 Child targets, no significant improvement was observed with $72 \%$ of the test ears still did not achieve the optimal fit-to-targets. When the RMS was recalculated at three frequencies $(500,1000$, and 2000 $\mathrm{Hz}$ ), excluding the $4000 \mathrm{~Hz}, 36 \%$ of the ears fitted with the prefit procedure deviated from the DSL v5 targets by more than $5 \mathrm{~dB}$ RMS. After adjusting the hearing aids to attempt to match with the DSL v5 Child targets, $25 \%$ of the test ears deviated from the DSL v5 Child targets by more than 5-dB RMS and failed to achieve the optimal fit-to-targets.

Table 1 shows the total number of fittings that failed to meet the DSL v5 Child targets at different frequencies and input levels. Numbers without brackets represent the ears before gain adjustment/prefit outputs

Table 1. The Total Number of Fittings that Fail to Meet the DSL v5 Prescribed Targets at Different Frequencies and Input Levels

\begin{tabular}{|c|c|c|c|c|c|c|}
\hline & & $250 \mathrm{~Hz}$ & $500 \mathrm{~Hz}$ & $1000 \mathrm{~Hz}$ & $2000 \mathrm{~Hz}$ & $4000 \mathrm{~Hz}$ \\
\hline \multirow[t]{2}{*}{50 input } & Fit-to-target $(+)$ & $4(3)$ & $0(0)$ & $0(0)$ & $29(0)$ & $12(0)$ \\
\hline & Fit-to-target $(-)$ & $0(0)$ & $1(0)$ & $3(2)$ & $0(3)$ & $40(40)$ \\
\hline \multirow[t]{2}{*}{65 input } & Fit-to-target $(+)$ & $0(0)$ & $0(0)$ & $0(0)$ & $1(0)$ & $0(0)$ \\
\hline & Fit-to-target $(-)$ & $5(3)$ & $13(9)$ & $18(14)$ & $13(12)$ & $40(40)$ \\
\hline \multirow[t]{2}{*}{75 input } & Fit-to-target (+) & $0(0)$ & $0(0)$ & $0(0)$ & $1(0)$ & $0(0)$ \\
\hline & Fit-to-target $(-)$ & $14(9)$ & $30(23)$ & $28(24)$ & $22(22)$ & $48(41)$ \\
\hline
\end{tabular}

Numbers without brackets represent the fittings/ears before gain adjustment/prefit output whereas numbers in bracket represents the fittings/ ears after gain adjustment to attempt to match with the DSLv5 prescribed targets. Fit-to-target $(+)$ means the output was higher than the prescribed outputs. Fit-to-target (-) means the output was lower than the prescribed outputs. 
whereas those in brackets represent the ears after gain adjustment. Fit-to-target $(+)$ shows that the output was higher than the prescribed output. Fit-to-target (-) means the opposite of $(+)$. The results showed that the prefit outputs were mostly below the DSL v5 Child prescribed outputs at all the test frequencies, for moderate- and high-input levels. Nonetheless, at low-input level, the prefit outputs were higher than the DSL v5 Child outputs at 250,2000, and $4000 \mathrm{~Hz}$. Twenty-nine ears at $2000 \mathrm{~Hz}$ and 12 ears at $4000 \mathrm{~Hz}$ had higher prefit outputs compared with prescribed outputs. It was ob- served that deviations of prefit outputs from DSL v5 Child outputs were most frequent at $4000 \mathrm{~Hz}$ and at high input levels. More than $80 \%$ of the ears failed to meet the DSL v5 Child outputs even after hearing aid adjustments at $4000 \mathrm{~Hz}$.

Figure $1 \mathrm{~A}$ and $\mathrm{B}$ show the manufacturer's prefit output versus the DSL v5 Child output at low and mid frequencies $(250,500$, and $1000 \mathrm{~Hz})$ and at high frequencies (2000 and $4000 \mathrm{~Hz}$ ) respectively, for different input levels and different hearing aid manufacturers. The diagonal line in the graph indicates identical prefit
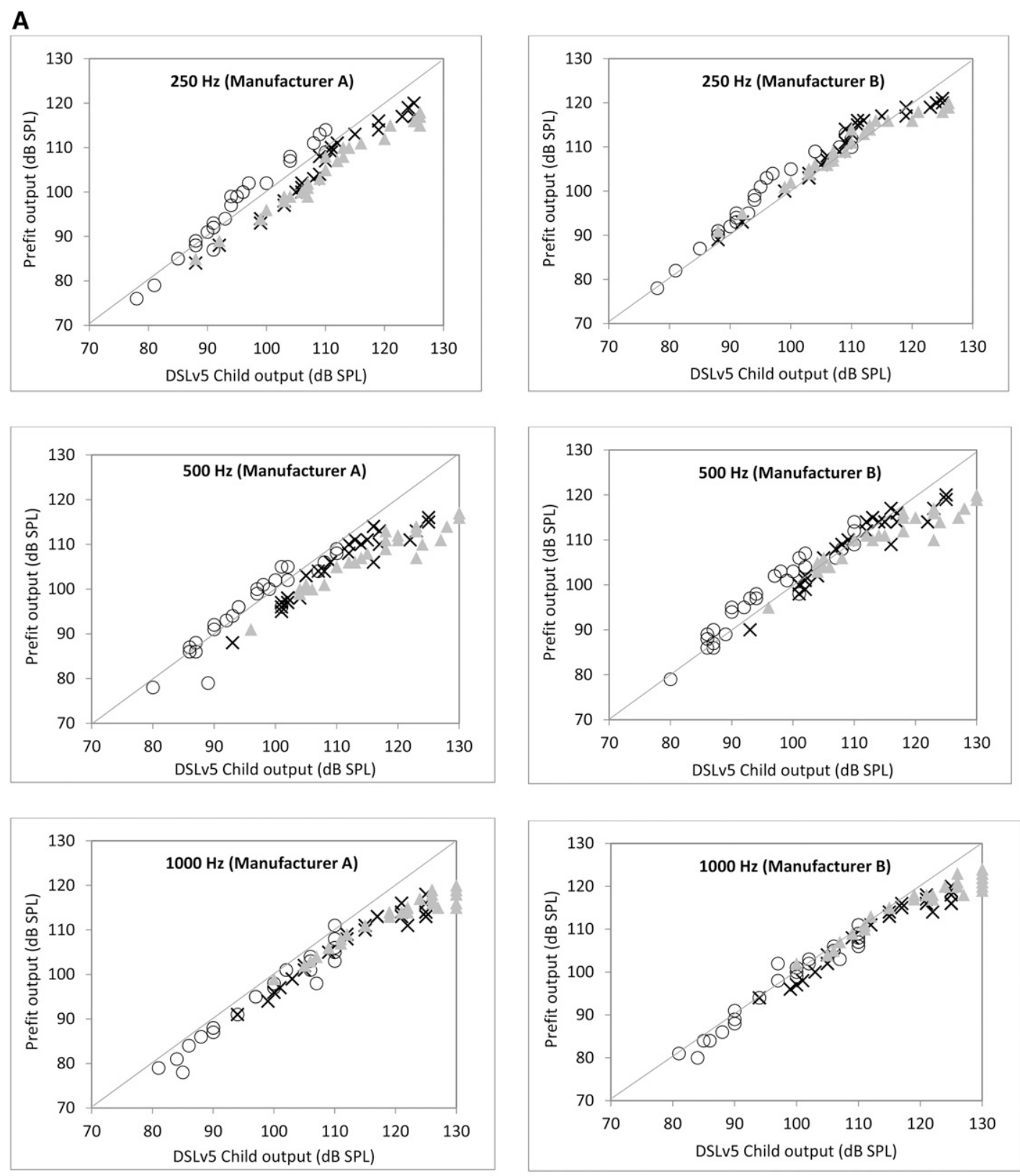

$0-50$ input level $x-65$ input level $A-75$ input level

Figure 1. (A) Different manufacturer prefit outputs as compared to DSL v5 Child outputs at low to mid frequencies (250, 500, and 1000 $\mathrm{Hz}$ ) and at different input levels. (B) Different manufacturer prefit outputs as compared to DSL v5 outputs at high frequencies (2000 and $4000 \mathrm{~Hz}$ ) and at different input levels. 
B
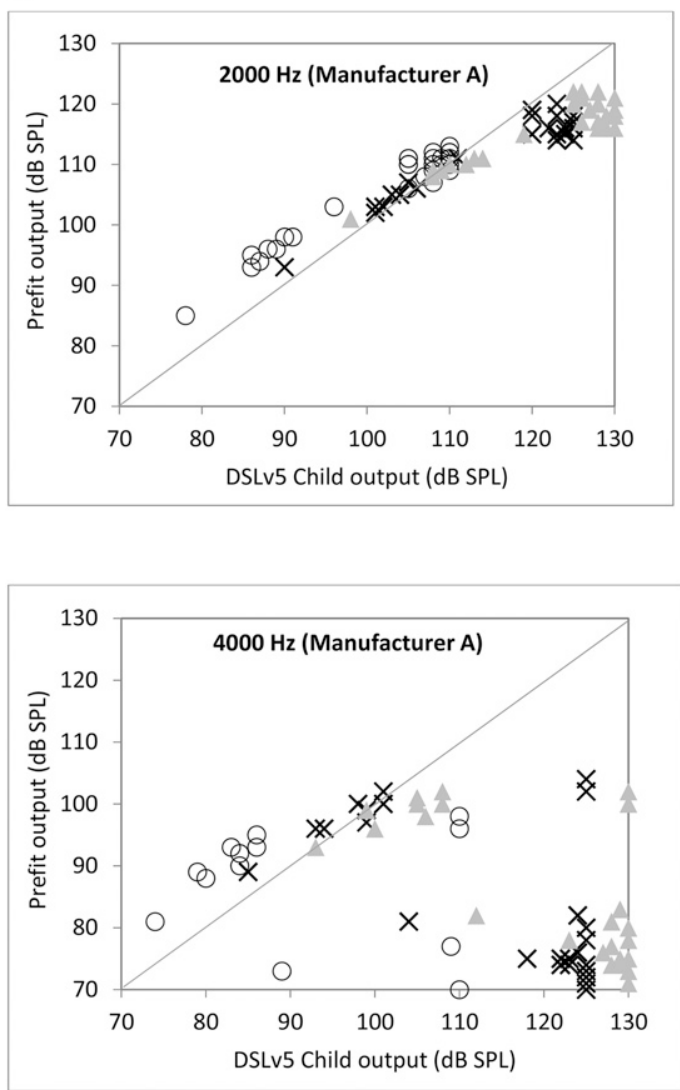

o-50 input level $x-65$ input level $\quad-75$ input level

Figure 1. (Continued).

output and DSL v5 Child output. Individual data that fall above the diagonal line means that the prefit output is higher than the DSL v5 Child output and vice versa for data that falls below the diagonal line.

Repeated measures analysis of variance (ANOVA) was carried out to investigate whether the manufacturer's prefit outputs differ significantly from the DSL v5 Child outputs for different hearing aids (Phonak or Unitron), input levels $(50,65$, or $75 \mathrm{~dB})$, and frequencies $(250$, $500,1000,2000$, and $4000 \mathrm{~Hz}$ ). The results showed that there was significant main effect of hearing aid $\left[F_{(1,27)}=\right.$ 264.36, $p<0.001]$, input level $\left[F_{(2,54)}=180.99, p<\right.$ $0.001]$, and also frequency $\left[F_{(4,108)}=52.57, p<0.001\right]$. Significant interactions were observed between hearing aid and input level $\left[F_{(2,54)}=54.46, p<0.001\right]$; hearing aid and frequency $\left[F_{(4,108)}=3.91, p<0.01\right]$ and input and frequency $\left[F_{(8,216)}=15.42, p<0.001\right]$. The three-way interaction between hearing aid, input level, and frequency was also significant $\left[F_{(8,216)}=10.03, p<0.001\right]$. Bonferroni test indicated that for Phonak hearing aid, the deviations of prefit outputs from DSL v5 Child outputs were significant between low and the other two input levels at all the frequencies. The deviations between moderateand high-input levels were not significant at 250 and
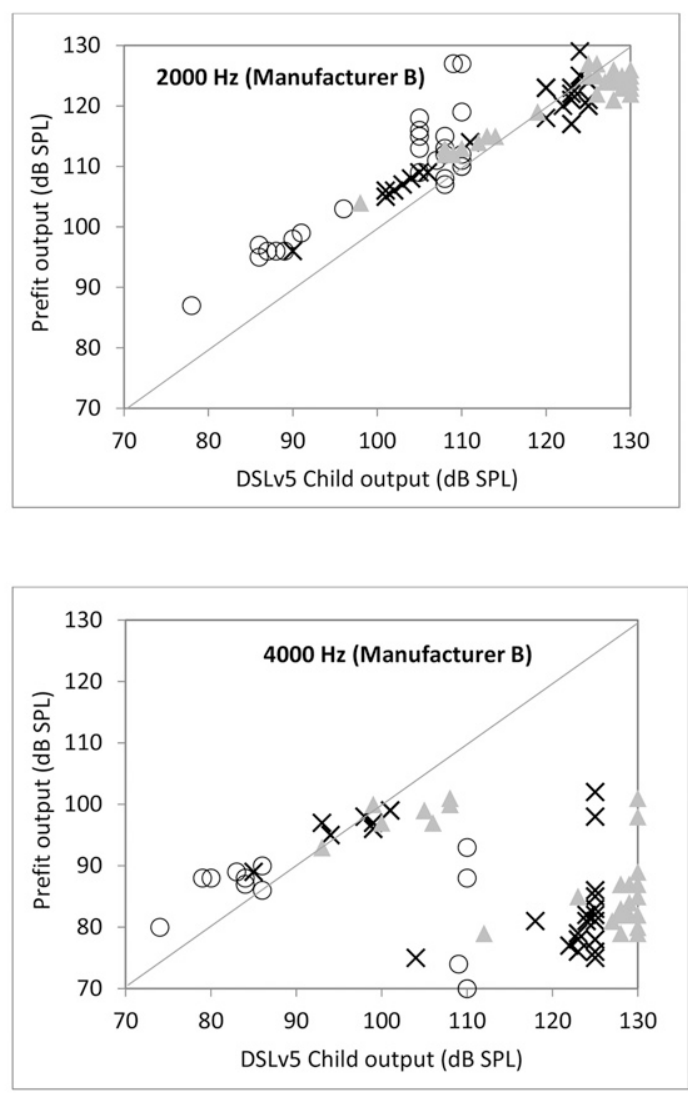

$1000 \mathrm{~Hz}$. By contrast, the deviations were significant between all input levels and frequencies for Unitron hearing aid. Furthermore, the prefit outputs were higher than the prescribed DSL v5 Child outputs at low-input level for both hearing aids at 250,500, and $2000 \mathrm{~Hz}$. At 1000 and $4000 \mathrm{~Hz}$, the prefit outputs were lower than the prescribed DSL v5 Child outputs at low-input level for both hearing aids.

\section{SII}

Generally, SII decreases with increased hearing loss. Thus, the SII values were analyzed according to different degree of loss. Figure 2 compares the mean SII values between the manufacturer's prefit output and the adjusted output for severe and profound hearing losses, at different input levels. Repeated measures ANOVA with SII as dependent variable and type of SII (SII for prefit and adjusted fitting), input level (50, 65 and $75 \mathrm{~dB}$ ), and hearing aid type (Phonak and Unitron) as independent variables indicated that the main effects of hearing aid type $\left[F_{(1,26)}=101.47, p<\right.$ $0.001]$ and input level $\left[F_{(2,52)}=56.04, p<0.001\right]$ were significant. Nevertheless, the type of SII was not 


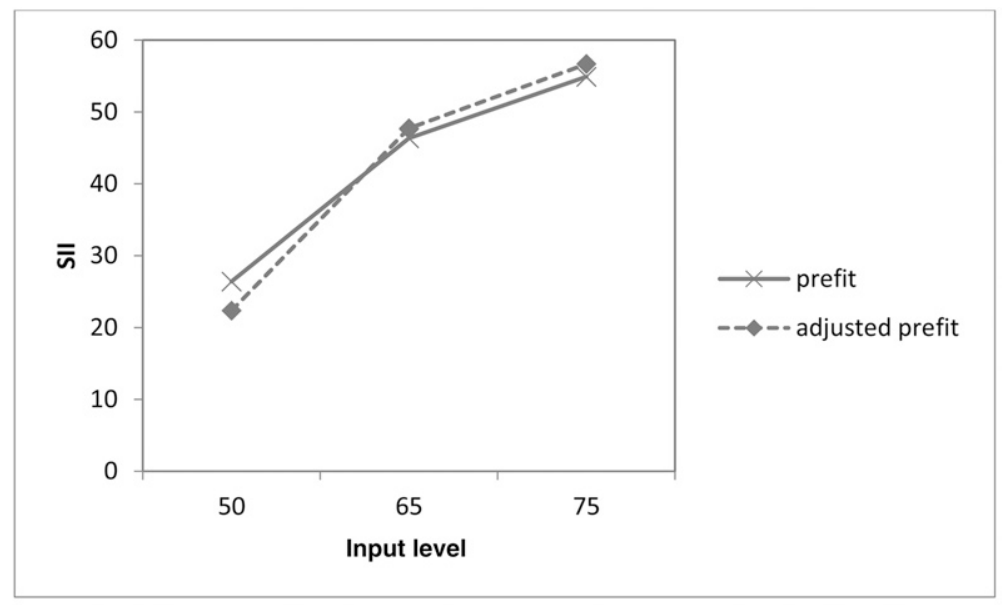

Severe loss

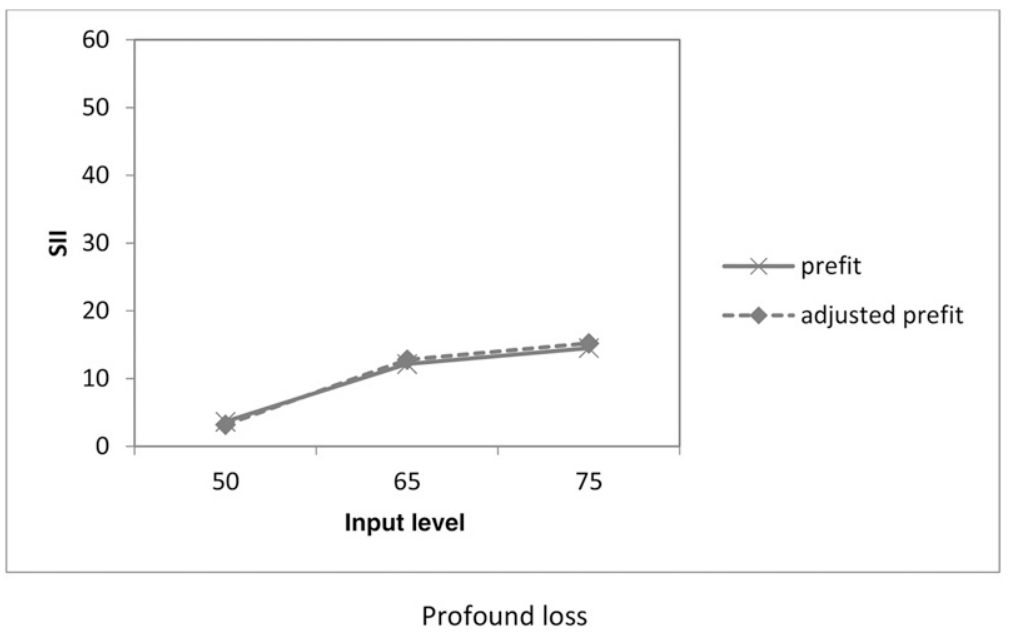

Figure 2. Mean SII (\%) for hearing aid before (prefit) and after hearing aid adjusted in attempt to meet the DSL v5 Child targets (adjusted prefit), at different input levels. Top panel shows results for severe hearing loss and bottom panel shows results for profound hearing loss.

significant $\left[F_{(1,26)}=0.66, p=0.42\right]$, suggesting that there were no significant differences between the SII of prefit output and adjusted output. Furthermore, there were no significant interactions between the hearing aid type, input level, and type of SII. As aforementioned, normative SII values based on the pure tone average for pediatric hearing aid fittings exist. Based on the Aided SII Normative Values (Bagatto et al, 2016), 68\% of the SII values that were generated for the manufacturer's prefit output and the output adjusted to match with DSL v5 Child targets fell below the lower confidence interval of the normative data (SII $<20 \%$ at low level and $<40 \%$ at moderate level).

\section{DISCUSSION}

$\mathrm{T}$ his study found that $73 \%$ of manufacturer's prefit failed to achieve the DSL v5 Child targets with RMS exceeding $5 \mathrm{~dB}$ at 500, 1000, 2000, and $4000 \mathrm{~Hz}$. The percentage was higher than the $64 \%$ reported in Aazh and Moore's study in adults (2007). Nevertheless, it should be noted that previous study used different pass/fail criterion ( $\pm 10 \mathrm{~dB}$ instead of $\pm 5 \mathrm{~dB}$ ) and the participants had better hearing thresholds. Furthermore, adjusting the hearing aids in an attempt to match the DSL v5 Child targets did nothing to improve the fit-totargets. Nonetheless, when $4000 \mathrm{~Hz}$ was excluded from RMS calculation, the percentage of ears that failed to achieve the DSL v5 Child targets decreased from $36 \%$ to $25 \%$ after hearing aid adjustments.

Moving on, the deviations of manufacturer's prefit outputs from DSL v5 Child outputs at individual frequencies and at different input levels were examined separately from each other. The individual data in Figure 1A and $\mathrm{B}$ revealed that the deviations were larger and more frequent in high frequencies. On average, the mean differences between the prefit and DSL v5 prescribed outputs were mostly within $\pm 5 \mathrm{~dB}$, except for $4000 \mathrm{~Hz}$ where the mean prefit outputs were below the prescriptive targets for all input levels. This agrees with previous 
studies on adults that revealed reasonable agreement between simulated and measured insertion gain between the frequency of 250 and $1000 \mathrm{~Hz}$ (Hawkins and Cook, 2003; Aarts and Caffee, 2005; Aazh and Moore, 2007). Even though the mean values suggested the existence of a reasonable agreement between the prefit and DSL v5 Child targets from 250 to $2000 \mathrm{~Hz}$, Table 1 demonstrated that for some fittings, the prefits still failed to meet the DSL v5 Child targets ( $>20 \%)$, especially at moderateand high-input levels. In these cases (except for one), the prefit outputs consistently fell below the prescriptive targets. At 50-dB input level however, about 7\%, 52\%, and $21 \%$ of the prefits were above the DSL v5 Child targets at 250,2000 , and $4000 \mathrm{~Hz}$, respectively.

ANOVA analyses showed a significant main effect of input levels. This suggested that deviations of manufacturer's prefit outputs from DSL v5 Child outputs were noticeably different between low-, moderate-, and high-input levels. The mean results suggested at low-input level, the prefit outputs were higher than the DSL v5 prescribed outputs and vice versa for moderateand high-input levels. Previous studies had suggested that there was a risk of hearing damage and loudness discomfort that was associated with high gains of hearing aid provided at moderate- and high-input levels to children (Ching et al, 2013; Quar et al, 2013). It is not clear if there is any effect of over-amplified soft sounds on auditory function, therefore further research in this aspect is required. The deviations of prefit output from DSL v5 Child output were not only dependent on the input level but also the frequency and types of hearing aids. The deviations were the largest (up to $33 \mathrm{~dB}$ on average) at $4000 \mathrm{~Hz}$ relative to other tested frequencies. Although prefit outputs significantly deviated from the DSL v5 Child outputs for different hearing aid, the mean differences were small (within $5 \mathrm{~dB}$ ) at each tested frequency.

Although the analysis of the RMS at three frequencies demonstrated some improvements on fit-to-targets (500, 1000 , and $2000 \mathrm{~Hz}$ ), there were no significant changes in the predicted speech perception or SII. This was still true even after the attempt to adjust the hearing aids to match with the DSL v5 Child targets. Also, more than $50 \%$ of the SII values calculated that were based on prefit output and adjusted output did not fall within the Aided SII Normative Values from Bagatto et al (2016). This was due to the under amplification at frequencies beyond $2000 \mathrm{~Hz}$. Furthermore, the deviations of the hearing aid output from the DSL v5 Child outputs remained high at $4000 \mathrm{~Hz}$ even after the gain of hearing aids had been adjusted. Previous studies demonstrated that even when verification with real-ear measures and electroacoustic measurements were carried out, prescriptive targets could sometimes not be matched by hearing aids at high frequencies. This was due to the limitations of the hearing aid itself (Ching et al, 2010; 2015; McCreery et al, 2013; Quar et al, 2013). After comparing the fit-to-targets achieved by a commercial hearing aid among children with moderately severe to profound hearing losses, Ching et al (2015) reported that although targets between 250 and $2000 \mathrm{~Hz}$ were well matched for both NAL-NL1 and DSL v5 prescriptions, gain at $4000 \mathrm{~Hz}$ were matched for NAL-NL1 only. This is because the DSL v5 Child procedure normally prescribes higher overall gain, especially for high-frequency region (Ching et al, 2015).

The impact of fit-to-targets on predicted speech performance may differ if different SII model was used. In relating audibility to speech performance, the NAL-NL1 procedure adopted a modified SII model that considers the level distortion factor and the HLD. The level distortion factor is related to the deterioration of the speech performance as a result of high SPLs received by the ear. This is due to the inability of the cochlea to analyze the signal when it is presented at a level in which even a normal cochlea cannot function optimally. The HLD refers to the decreased ability of the damaged cochlea to extract information even when it is audible (Ching et al, 1998; 2001). Ching et al (2015) reported that on average, the SII was lower for NAL-NL1 than for DSL v5 prescription at low input level. Lower SII and possible better fit-to-targets achieved by NAL-NL1 procedure may affect the conclusions made in this study.

The underachieved amplification at high frequencies and the relatively low SII values for hearing aids fitted with prefit procedure alone and adjusted to attempt to match to the prescriptive targets suggested a different amplification option such as a cochlear implant is necessary to improve patients' auditory performance. Although the benefits of cochlear implants are well documented, this technology is still "unreachable" to many children with severe and profound hearing losses. This is especially the case in developing countries in which only $1 \%$ of the people who would benefit from cochlear implants, received one (Janssen, 2016). Financial constraints, public awareness, and availability of intervention centers and qualified cochlear implant professionals were among the factors that limit the accessibility of cochlear implants in many developing countries (McPherson and Brouillette, 2008). Today, many hearing aid manufacturers offer some algorithms to improve access to high-frequency information, generally known as FL. The aim of FL hearing aids is to deliver high-frequency information to a lower frequency region, where hearing thresholds are potentially better. Alexander (2013) review stated that children might experience greater benefit from FL compared with adults because children appear to have greater "deficit" when identifying speech under identical conditions. In other words, in conditions where adults are performing near the ceiling of their abilities using conventional amplification, children might still be able to benefit from additional information gained via frequency-lowered speech (Alexander, 2013).

Apart from that, using an adapted SII that takes into account FL strategy, McCreery et al (2014) found 
improved speech audibility and also speech recognition in adults and children fitted with FL strategy relative to conventional amplification. Whereas the benefits of FL to children are not fully known yet, the current AAA (2013) amplification protocol suggests that the possibility of employing FL in children should be determined individually. Also, electroacoustic verification should be performed to fine tune the FL settings for all candidates. Moreover, detailed descriptions on the assessment of candidacy and verification procedure for FL can be found in a study by Glista et al (2016) and Scollie et al (2016). They recommended the use of prerecorded and calibrated stimuli, the fricatives $/ \mathrm{s} /$ and $/ / /$ to determine the audibility of the high-frequency sounds and to adjust the strength of the FL setting. These recommendations had been incorporated into the latest version of the Audioscan system, Audioscan ${ }^{\circledR}$ Verifit2. The stimuli were incorporated to overcome the limitations of previous modified speech stimuli, which comprised bands of high-frequency speech energy at specific center frequencies: $3150,4000,5000$, and $6300 \mathrm{~Hz}$. This shows that in cases where FL technology is fitted, the use of verification procedures specifically for FL is necessary to provide optimum fitting.

\section{LIMITATIONS OF STUDY}

$\mathrm{T}$ he accuracy of manufacturer's prefit procedure to match with the DSL v5 Child targets was only investigated at low-, moderate-, and high-input levels. The saturated SPL of the hearing aids was not examined and should be included in future study. Furthermore, saturated SPL is often associated with sound discomfort, sound quality, and acceptance of hearing device. This should be regarded as an important electroacoustic parameter during verification procedure. In this study, coupler measures were used to compare the prefit outputs with the outputs prescribed DSL v5 Child. This resulted in an unrealistic scenario because coupler provides more occluding than earmold coupling and hence the match-to-target may not be achievable in the actual ears. Also, the information input into the programming software was "13 tubing, occluded earmold and no vent" which was in contrast to the coupler and insert-earphones measures conducted in the actual study. The misrepresentation of information on the coupling strategy may have an impact on the gain prescribed by the software.

\section{CONCLUSIONS}

$\mathrm{O}$ $\mathrm{n}$ average, hearing aid output generated by the manufacturer's prefit procedure has a reasonably good agreement with the DSL v5 Child targets at low and mid frequencies. This is at least true for the two hearing aids investigated in the study. Optimal fit-to-targets improved slightly for individual ears after the hearing aids had been adjusted, and the SII did not improve. The deviations of hearing aid output from DSL v5 Child targets at
$4000 \mathrm{~Hz}$ suggest an inadequate access to high frequency sounds. In such cases, other strategies such as cochlear implantation or fittings that incorporate advanced hearing aid features such as FL should be considered.

Acknowledgments. The study was supported by the Young Investigator Grant Universiti Kebangsaan Malaysia (UKM) awarded to the first author (GGPM-2014-053). The authors gratefully acknowledge the support and participations of the children and their families in this study.

\section{REFERENCES}

Aarts NL, Caffee CS. (2005) Manufacturer predicted and measured REAR values in adult hearing aid fitting: accuracy and clinical usefulness. Int J Audiol 44(5):293-301.

Aazh H, Moore BCJ. (2007) The value of routine real ear measurement of the gain of digital hearing aids. J Am Acad Audiol 18(8): 653-664.

Alexander JM. (2013) Individual variability in recognition of frequency-lowered speech. Semin Hear 34(2):86-109.

American Academy of Audiology (AAA). (2013) American Academy of Audiology Clinical Practice Guidelines on pediatric amplification. http://www.audiology.org/resources/documentlibrary/Documents/ PediatricAmplificationGuidelines.pdf.

Audioscan. (2014) Verifit User's Guide 3.10. Ontario, Canada: Etymonic Design Inc.

Bagatto M, Moodie S, Brown C, Malandrino A, Richert F, Clench D, Scollie S. (2016) Prescribing and verifying hearing aids applying the American Academy of Audiology pediatric amplification guideline: protocols and outcomes from the Ontario infant hearing program. J Am Acad Audiol 27(3):188-203.

Bagatto M, Moodie S, Scollie S, Seewald R, Moodie S, Pumford J, Liu KP. (2005) Clinical protocols for hearing instrument fitting in the desired sensation level method. Trends Amplif 9(4):199-226.

Bagatto MP. (2001) Optimizing your RECD measurements. Hear J 54(9):32-36.

Bagatto MP, Seewald RC, Scollie SD, Tharpe AM. (2006) Evaluation of a probe-tube insertion technique for measuring the realear-to-coupler difference (RECD) in young infants. $J$ Am Acad Audiol 17(8):573-581.

Byrne D. (1983) Theoretical Prescriptive Approaches to Selecting the Gain and Frequency Response of a Hearing Aid. Monographs in Contemporary Audiology. Upper Darby, PA: Educational Services Division of Instrumentation Associates.

Ching TYC, Dillon H, Byrne D. (1998) Speech recognition of hearingimpaired listeners: predictions from audibility and the limited role of high-frequency amplification. J Acoust Soc Am 103(2):1128-1140.

Ching TYC, Dillon H, Katsch R, Byrne D. (2001) Maximizing effective audibility in hearing aid fitting. Ear Hear 22(3):212-224.

Ching TYC, Johnson EE, Seeto M, Macrae JH. (2013) Hearing-aid safety: a comparison of estimated threshold shifts for gains recommended by NAL-NL2 and DSL $\mathrm{m}[\mathrm{i} / \mathrm{o}]$ prescriptions for children. Int J Audiol 52(Suppl 2):S39-S45.

Ching TYC, Quar TK, Johnson EE, Newall P, Sharma M. (2015) Comparing NAL-NL1 and DSL v5 in hearing aids fit to children 
with severe or profound hearing loss: goodness of fit-to-targets, impacts on predicted loudness and speech intelligibility. J Am Acad Audiol 26(3):260-274.

Ching TYC, Scollie SD, Dillon H, Seewald R. (2010) A cross-over, double-blind comparison of the NAL-NL1 and the DSL v4.1 prescriptions for children with mild to moderately severe hearing loss. Int $J$ Audiol 49(Suppl 1):S4-S15.

Davidson LS, Skinner MW. (2006) Audibility and speech perception of children using wide dynamic range compression hearing AIDS. Am J Audiol 15(2):141-153.

Glista D, Hawkins M, Scollie SD. (2016) An Update on Modified Verification Approaches for Frequency Lowering Devices. AudiologyOnline.com, May.

Hawkins DB, Cook JA. (2003) Hearing aid software predictive gain values: how accurate are they? Hear $J$ 56(7):26-34.

Janssen J. (2016) Are cochlear implants utilized worldwide? http://www.audiologyonline.com/ask-the-experts/are-cochlearimplants-utilized-worldwide-18721. Retrieved March 9, 2017.

McCreery RW, Alexander J, Brennan MA, Hoover B, Kopun J, Stelmachowicz PG. (2014) The influence of audibility on speech recognition with nonlinear frequency compression for children and adults with hearing loss. Ear Hear 35(4):440-447.

McCreery RW, Bentler RA, Roush PA. (2013) Characteristics of hearing aid fittings in infants and young children. Ear Hear 34(6):701-710.

McCreery RW, Walker EA, Spratford M, Bentler R, Holte L, Roush P, Oleson J, Van Buren J, Moeller MP. (2015) Longitudinal predictors of aided speech audibility in infants and children. Ear Hear 36(Suppl 1):24S-37S.

McPherson B, Brouillette R. (2008)Audiology in Developing Countries: Introduction. New York, NY: Nova Science Publisher, Inc.

Moodie S, Pietrobon J, Rall E, Lindley G, Eiten L, Gordey D, Davidson L, Moodie KS, Bagatto M, Haluschak MM, Folkeard P, Scollie S. (2016) Using the real-ear-to-coupler difference within the American Academy of Audiology Pediatric Amplification Guideline: protocols for applying and predicting earmold RECDs. J Am Acad Audiol 27(3):264-275.

Moodie KS, Seewald RC, Sinclair ST. (1994) Procedure for predicting real-ear hearing aid performance in young children. $A m J$ Audiol 3:23-30.

Moore BC, Glasberg BR. (1997) A model of loudness perception applied to cochlear hearing loss. Aud Neurosci 3:289-311.

Moeller MP, Tomblin JB; OCHL Collaboration. (2015) Epilogue: conclusions and implications for research and practice. Ear Hear 36(Suppl 1):92S-98S.

Mueller HG. (2001) Probe microphone measurements: 20 years of progress. Trends Amplif 5(2):35-68.

Mueller HG. (2005a) Fitting hearing aids to adults using prescriptive methods: an evidence-based review of effectiveness. $J A m$ Acad Audiol 16(7):448-460.
Mueller HG. (2005b) Probe-mic measures: hearing aid fitting's most neglected element. Hear J 58(10):21-30.

Mueller HG, Picou EM. (2010) Survey examines popularity of realear probe microphone measures. Hear $J$ 63(5):27-32.

Munro KJ, Hatton N. (2000) Customized acoustic transform functions and their accuracy at predicting real-ear hearing aid performance. Ear Hear 21(1):59-69.

Pavlovic CV, Studebaker GA, Sherbecoe RL. (1986) An articulation index based procedure for predicting the speech recognition performance of hearing-impaired individuals. J Acoust Soc Am 80(1):50-57.

Quar TK, Ching TYC, Newall P, Sharma M. (2013) Evaluation of real-world preferences and performance of hearing aids fitted according to the NAL-NL1 and DSL v5 procedures in children with moderately severe to profound hearing loss. Int $J$ Audiol 52(5):322-332.

Scollie SD. (2008) Children's speech recognition scores: the speech intelligibility index and proficiency factors for age and hearing level. Ear Hear 29(4):543-556.

Scollie S, Glista D, Seto J, Dunn A, Schuett B, Hawkins M, Pourmand N, Parsa V. (2016) Fitting frequency-lowering signal processing applying the American Academy of Audiology pediatric amplification guideline: updates and protocols. J Am Acad Audiol 27(3):219-236.

Scollie S, Seewald R, Cornelisse L, Moodie S, Bagatto M, Laurnagaray D, Beaulac S, Pumford J. (2005) The desired sensation level multistage input/output algorithm. Trends Amplif 9(4):159-197.

Seewald RC. (1991) Hearing aid output limiting considerations for children. In: Feigin JA, Stelmachowicz PG, eds. Pediatric Amplification: Proceedings of the 1991 National Conference. Omaha, NE: Boy's Town National Research Hospital Press, 19-36.

Seewald RC, Mills J, Bagatto MP, Scollie SD, Moodie S. (2008) A comparison of manufacturer-specific prescriptive procedures for infants. Hear J 61(11):26-34.

Seewald RC, Moodie KS, Sinclair ST, Scollie SD. (1999) Predictive validity of a procedure for pediatric hearing instrument fitting. Am J Audiol 8(2):143-152.

Seewald RC, Ross M, Spiro MK. (1985) Selecting amplification characteristics for young hearing-impaired children. Ear Hear 6(1):48-53.

Sinclair ST, Beauchaine KL, Moodie KS, Feigin JA, Seewald RC, Stelmachowicz PG. (1996) Repeatability of a real-ear-to-coupler difference measurement as a function of age. Am J Audiol 5:52-56.

Stiles DJ, Bentler RA, McGregor KK. (2012) The speech intelligibility index and the pure-tone average as predictors of lexical ability in children fit with hearing AIDS. J Speech Lang Hear Res 55(3):764-778.

Studebaker GA, Sherbecoe RL, McDaniel DM, Gray GA. (1997) Age-related changes in monosyllabic word recognition performance when audibility is held constant. J Am Acad Audiol 8(3):150-162.

Tomblin JB, Harrison M, Ambrose SE, Walker EA, Oleson JJ, Moeller MP. (2015) Language outcomes in young children with mild to severe hearing loss. Ear Hear 36(Suppl 1):76S-91S. 